\title{
Gas-distension, double-contrast duodenography using the Scott-Harden gastroduodenal tube
}

\author{
SILVANO RAIA AND LOUIS KREEL \\ From the Departments of Medicine and Radiology \\ the Royal Free Hospital, London
}

Hypotonic duodenography is a well recognized and well established procedure (Mallet-Guy and Jacquemet, 1963), by which the duodenal loop is outlined in a flaccid state. Variations of contour due to inherent duodenal movement are thus overcome. A constant pattern of the inner wall of the duodenal mucosa is obtained unobscured by overlying stomach, and even minor distortions become visible. Lesions of the pancreas and papilla of Vater affecting the duodenal loop can be distinguished from distortion due to peristalsis.

'Duodenographie hypotonique' (atonic duodenography) was performed using a double-contrast technique. To improve the technique it was considered imperative to produce marked distension of the duodenal loop as this would give the maximum amount of information as to alterations in the duodenal mucosa. It is considered that the distension of the duodenal lumen will force the inner wall of the duodenal loop against the juxtaposed pancreas, producing a constant appearance, and thus minor alteration due to early lesions of pancreatic tissue will become visible. By this method not only can contiguous changes producing filling defects be shown, but also minor alterations in the duodenal mucosa itself. By using the double-lumen gastroduodenal tube (Scott-Harden, 1960) and giving the oxyphenonium intravenously, we are able to perform this procedure, in the average case, in 15 minutes.

\section{TECHNIQUE}

Quarter of an hour before the commencement of the procedure the patient is given an amethocaine lozenge to suck. A double-lumen gastro-duodenal tube (ScottHarden) is prepared by lubricating the outer tube with xylocaine gel and the back of the patient's mouth is sprayed with a few puffs of $4 \%$ xylocaine.

The tube is then passed to just beyond the gastrooesophageal junction with the patient sitting in a chair. The patient is then moved to the screening table and in the erect position the inner tube is pushed on for a further four inches, after which both tubes are passed on together until the tip of the inner tube reaches the position of the pyloric canal.

A mixture of $16 \mathrm{ml}$. of Micropaque and $4 \mathrm{ml}$. of water is then injected down the inner tube to visualize it. Only a minimal amount of this barium mixture is allowed to escape into the stomach so as not subsequently to obscure the duodenum. If the exact position of the pylorus can be demonstrated with the minimal injection of barium, it is very helpful in passing the inner tube through the pyloric canal. While an assistant slowly passes both tubes on together, the radiologist can guide the inner tube through the pyloric canal by external pressure on the lower part of the greater curve of the stomach. The inner tube is passed on to the second part of the duodenum by the assistant moving both tubes together until the distal end of the outer tube reaches the pyloric canal. At this stage it is often advisable for the outer tube to be pulled back a little to relieve any tension on the fundus and body of the stomach.

The screening table is tilted to the horizontal position and the patient is then given $4 \mathrm{mg}$. of oxyphenonium ${ }^{1}$ intravenously. The oxyphenonium is given at this stage rather than at the start of the procedure as it has been shown that loss of gastric tonus makes it more difficult to pass the tube through the pyloric canal (Kreel, 1965). With the patient in the horizontal position and the left shoulder somewhat raised, the remainder of the barium mixture is slowly injected at the same time as the oxyphenonium is being given intravenously. This usually causes quite marked churning movements of the second part of the duodenum and helps to coat the duodenal mucosa with an adherent film of Micropaque. This is a further reason for delaying the injection of oxyphenonium until after the inner tube is in position in the second part of the duodenum. With the patient in the horizontal position with the left side raised, very little of the contrast medium is propelled onwards in spite of the initial peristaltic activity.

Following the slow injection of the barium, xylocaine ( $2 \mathrm{ml}$. of $4 \%$ diluted to $10 \mathrm{ml}$.) is slowly injected into the second part of the duodenum. This further helps to produce relaxation of the duodenal loop. Approximately three minutes after the intravenous injection of the oxyphenonium the duodenal loop can be seen to become flaccid and immobile on fluoroscopy. The patient is then

${ }^{1}$ Antrenyl (Ciba). 
placed quite flat on the back and warned that there will be momentary discomfort with the oxygen distension.

The oxygen cylinder is turned on to run at 8 to $10 \cdot 1 / \mathrm{min}$. At the command, the assistant connects the well-fitting end of the oxygen tube to the Scott-Harden tube. The duodenal loop is seen to distend. A film is taken and the oxygen is immediately disconnected from the duodenal tube. Forced oxygen insufflation is present for 3 to 4 seconds only. The timing of the forced oxygen insufflation is dependant on the appearance of atonicity of the duodenal loop. It is imperative to observe the duodenal loop and be quite sure that all spontaneous movements have ceased before the oxygen insufflation is begun.

The same procedure is repeated in each oblique position and a final film is again taken with the patient flat on his back. Four films of the duodenal loop are usually sufficient. Short exposure times in the order of 0.03 to 0.06 second give the best results, using a kilovoltage of about 95 and milliamperage of 300 on an average patient with fast screens ${ }^{1}$ and films ${ }^{2}$.

With a well-coordinated team, in the average patient the whole procedure can be performed within 15 minutes.

\section{DISCUSSION}

Following the experience of continental workers, we at first administered the oxyphenonium by intramuscular injection. However, it was soon clear that most of the time taken up by the procedure was spent in waiting for the duodenal loop to become

${ }^{2}$ IIford fast tungstate screen.

${ }^{3}$ Kodak blue brand

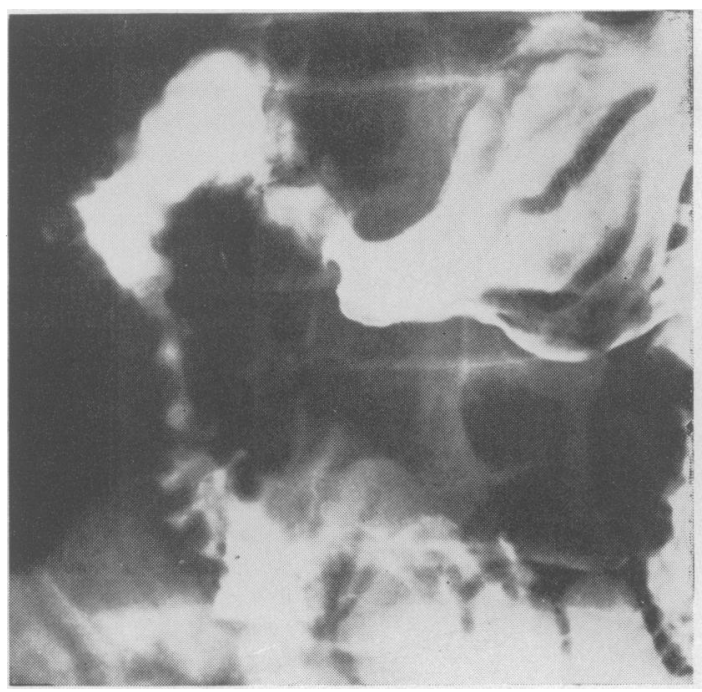

quiescent. Sometimes this would take up to 12 minutes. On administration of 4 to $6 \mathrm{mg}$. oxyphenonium by the intravenous route the atonic state of the duodenum is produced in two to three minutes, and the same dose appears to have a more marked effect. Less oxygen insufflation is required and often the final supine film can be taken without further gas distension. Moreover, patients appear to be much less affected by the gas distension of the duodenum following the intravenous administration of oxyphenonium than by the intramuscular route. We have also tried using Buscopan intravenously, but its action is too transient to be completely effective in this procedure.

It has been found that undiluted Micropaque is somewhat too viscous for injection down the inner tube of the double-lumen gastroduodenal tube. By the slight dilution with water as previously indicated, the barium can easily be injected and dilution does not interfere with its ability to adhere to the duodenal mucosa. We have tried using a water-soluble iodine-containing contrast medium (e.g., Gastrografin), but this provokes marked peristalsis of the duodenal loop.

To achieve distension and double-contrast visualization of the duodenal loop, the tip of the inner tube must be in the second part of the duodenum, and oxygen is insufflated at 8 to $10 \mathrm{l} / \mathrm{min}$. until optimum distension is obtained. The usual irregular duodenal pattern is converted to a regular pattern

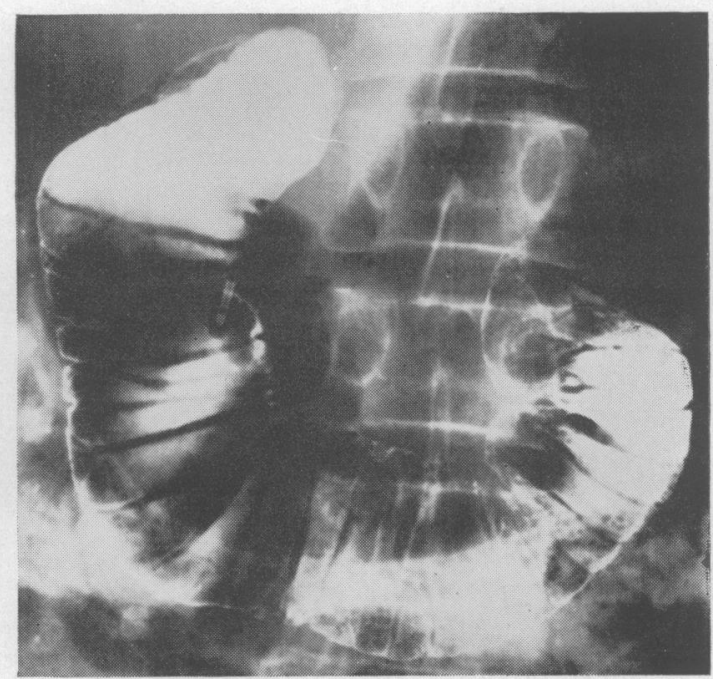

FIG. 1. The duodenal pattern as shown on a conventional barium meal is compared with that obtained on gas-distension duodenography. This patient had a malabsorption syndrome and it was considered important to exclude any evidence of chronic pancreatitis. On gas-distension duodenography it can be seen that the tranvserse duodenal folds are intact and run from the lateral to the medial margins. Note also the slight bulge of the duodenum in the distal half of the second part on the medial margin. These findings indicate a normal duodenal loop. 


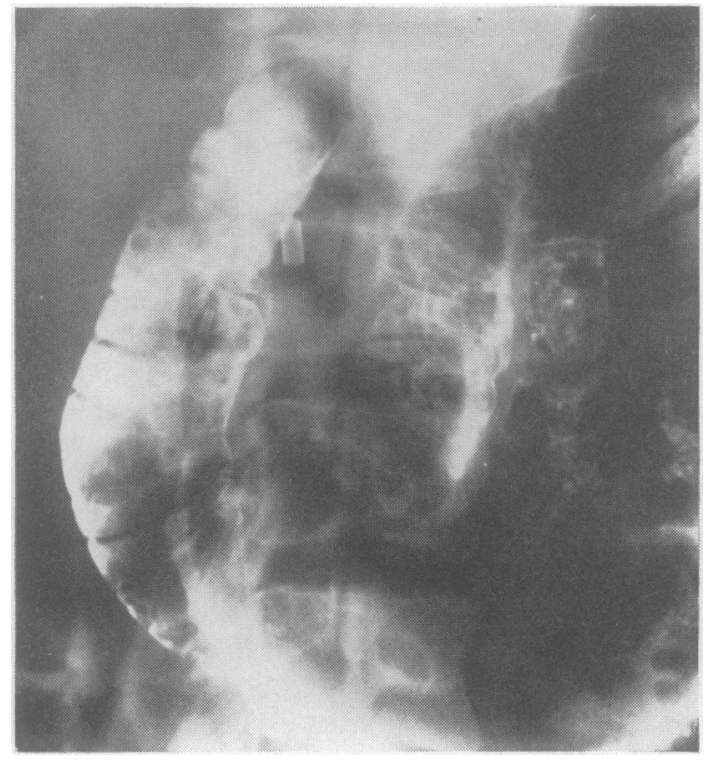

FIG. 2. Gas-distension duodenograph in a case of chronic pancreatitis. The medial margins of the middle folds of the second part of the duodenum have become obliterated and the medial edge of the duodenum in this region is straight and does not produce the normal convex margin. of transverse folds. By direct fluoroscopic control, especially using a television monitor, the exact moment of maximum distension can be appreciated and the appropriate film taken. This requires teamwork. Both the assistant controlling the oxygen insufflation and the radiologist must be able to view the duodenal loop. Immediately the film is taken the oxygen insufflation is stopped, so that there is no possibility of overdistension. Provided the patient is told what to expect, little discomfort and reaction occur, especially if the oxyphenonium is given intravenously.

The normal duodenal mucosal pattern has an irregular margin where the barium lies in the depths of the folds. Seen en face, this produces a feathery pattern with lines running in all directions. These contours and folds tend to vary from film to film, due to the inherent movements that occur. This pattern differs markedly from that seen in gasdistension, double-contrast duodenography (Fig. 1). The margin becomes perfectly smooth and regular with indentations at the site of the transverse folds. The transverse mucosal bars run from margin to margin spaced 0.5 to $1 \mathrm{~cm}$. apart. Each lateral indentation corresponds to a fold and has its accompanying medial indentation. With this technique the pattern is constant from film to film. The
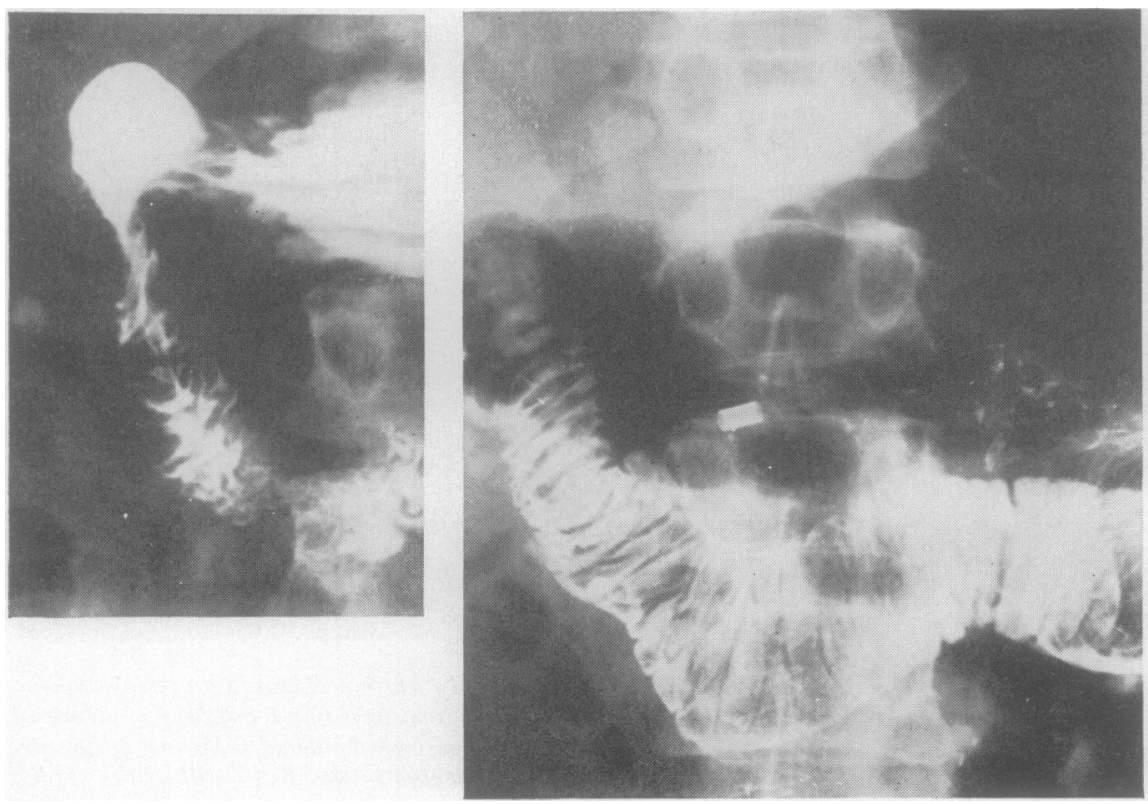

FIG. 3. Conventional barium meal film is compared with gasdistension duodenography. This patient had a sphincterotomy of the sphincter of Oddi. The operation was complicated by retroperitoneal and probably periduodenal infection. On the gas duodenography film the proximal half of the second part of the duodenum is shown to be narrowed and there is a sudden change in calibre of the duodenum corresponding to the incision of the duodenum. The bile ducts are also outlined by gas and can be seen above the gas in the stomach. 


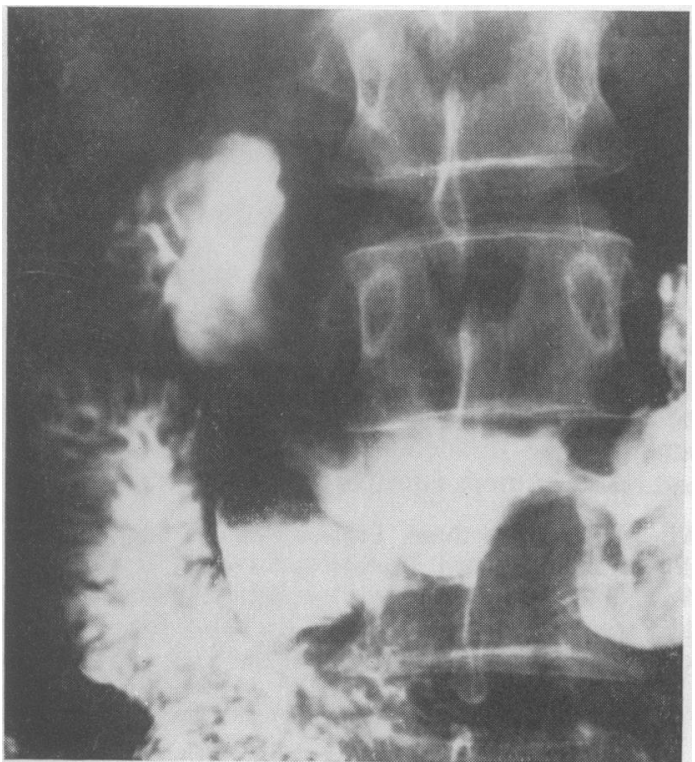

FIG. 4a.

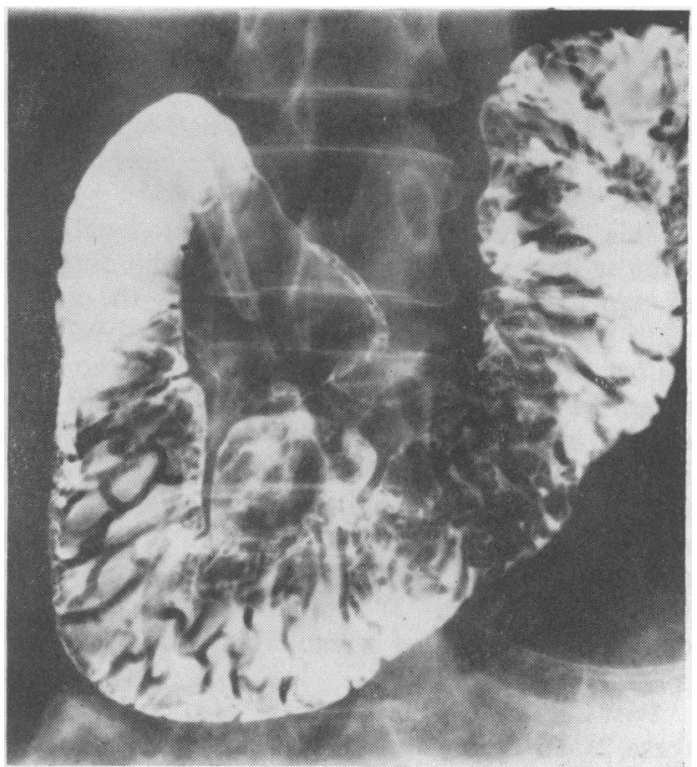

FIG. $4 \mathrm{~b}$.

FIG. 4a. Conventional barium meal examination shows a duodenal diverticulum arising from the third part. No detail is visible within the diverticulum.

FIG. 4b. The diverticulum is demonstrated by gas-distension duodenography. A barium fleck with radiating lines is visible. At operation this was shown to correspond to an ulcer within the diverticulum.

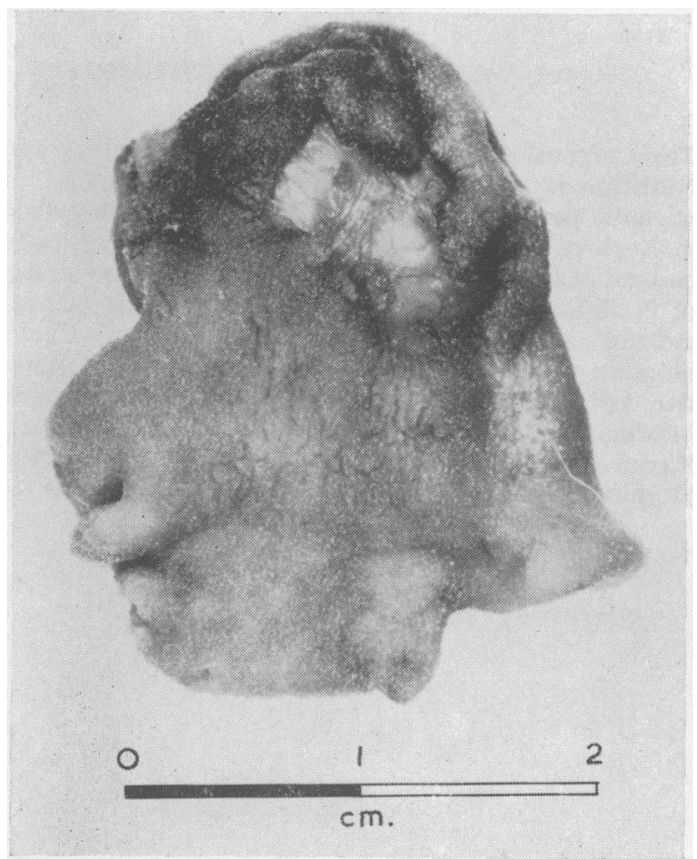

FIG. 4c.

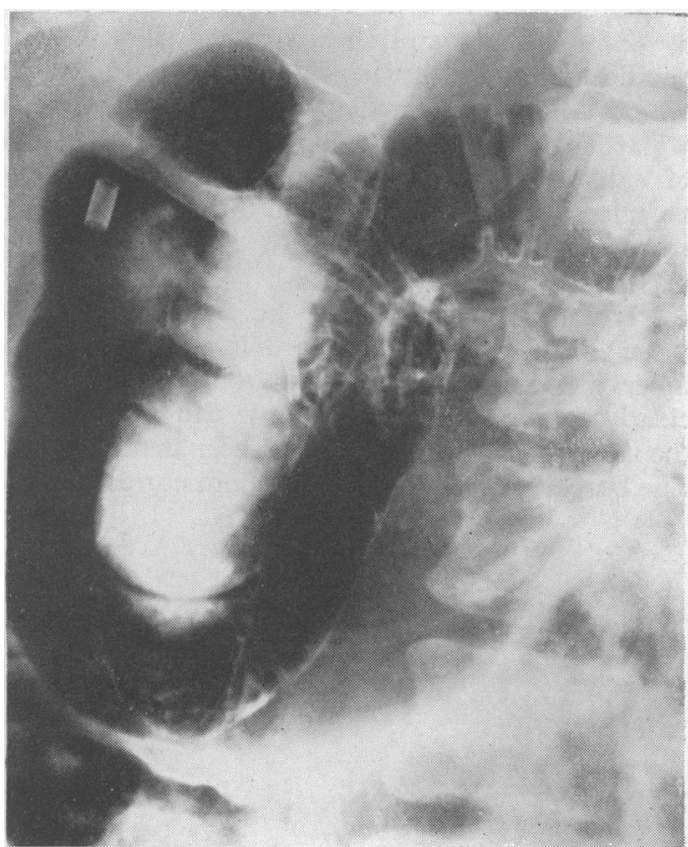

FIG. 5 .

FIG. 4c. The surgical specimen of the diverticulum showing the ulcer.

FIG. 5. The retro-duodenal space can be well shown in this procedure as the stomach contains gas. In the conventional barium meal this space tends to be obscured by the barium in the stomach. 
proximal half of the second part of the duodenum is a little narrower than the distal half which has a definite convex margin towards the normal pancreatic tissue.

This emphasis on the transverse duodenal folds is most important in connexion with the diagnosis of pancreatitis. In chronic pancreatitis the medial wall of the duodenum becomes involved and causes a loss of the transverse folds on the inner aspect of the duodenal loop and an irregular sawtoothed appearance of the medial margin (Fig. 2). Later the lumen is distinctly narrowed, which shows itself particularly in loss of the normal convexity of the medial margin of the distal half of the second part. Still later in the disease this may produce the Frostberg inverted ' 3 ' sign and mimic neoplastic changes with marked irregularity and indentation of the medial margin.

Post-operative changes can also be detected (case 2). In this particular instance sphincterotomy led to visualization of the common bile duct by air. Carcinoma of the head of the pancreas can be detected at a much earlier stage. The irregular duodenal margin, the loss of folds, and the indentation of the medial part of the duodenal loop are easily detected, and the appearances are moreover constant.

Lesions within the duodenal loop are unobscured by dense barium and shown in the same way as papillomas and neoplasms in the colon, when a double-contrast technique is used. This examination is particularly applicable to tumours of the papilla of Vater. Without going into any detail, it may also be mentioned that the retro-duodenal space is particularly well shown, as it is unobscured by barium in the stomach (Fig. 3).

Even small diverticula can be demonstrated, but more important in this regard is the fact that the inner aspect and contents of a diverticulum are clearly shown (Fig. 4b) where it can be seen that the ulcer in the diverticulum has been demonstrated. The barium fleck in the diverticulum corresponds to the ulcer seen on the surgical specimen (Fig. 4c). It is also hoped that the rare carcinoma of a diverticulum will now be capable of diagnosis.

\section{SUMMARY AND CONCLUSIONS}

A new method for the examination of the duodenum, using gas distension with atonicity, is presented. Some of its uses and the normal and abnormal appearances are briefly mentioned. It is hoped that by using this method lesions of the head of the pancreas and duodenum will be diagnosed earlier and result in more effective treatment.

We should like to thank Professor Sheila Sherlock and clinicians of the Royal Free Hospital for referring cases. We should also like to thank Mr. Gilson and members of the Photographic Department of the Royal Free Hospital for preparing the illustrations.

\section{REFERENCES}

Harden, W. G. Scott (1960). In Modern Trends in Diagnostic Radiology, edited by J. W. McLaren, 3rd series, pp. 84-87. Butterworth, London.

Kreel, L. (1965). Unpublished.

Mallet-Guy, P., and Jacquemet, P. (1963). Resultats de 500 Cas de duodenographie Hypotonique. J. Radiol. Electrol., 44, 249-58.

\section{NOTICE}

\section{CZECHOSLOVAKIAN SOCIETY OF GASTROENTEROLOGY} AND NUTRITION

The Czechoslovakian Society of Gastroenterology and Nutrition is preparing two international symposia of scientific papers to be held on 24 and 25 October 1966 in Karlovy Vary, Carlsbad, Czechoslovakia. Papers to be read at the first symposium, under the Chairmanship of Professor K. Herfort, will be on 'Rare forms of pancreatitis', and at the second symposium, under the Chairmanship of Dr. Z. Mařatka, the papers will be on 'Ulcerative colitis'. Applications and requests for further information should be sent to either Professor K. Herfort, Karlovo nám. 32, Prague 2, or to Dr. Z. Mařatka, Hospital Bulovka, Prague 8. 